\title{
Palliative care and prognosis in COPD: a systematic review with a validation cohort
}

\author{
This article was published in the following Dove Press journal: \\ International Journal of COPD \\ 9 June 2017 \\ Number of times this article has been viewed
}

\author{
Pere Almagro' \\ Sergi Yun' \\ Ana Sangil' \\ Mónica Rodríguez- \\ Carballeira' \\ Meritxell Marine' \\ Pedro Landete ${ }^{2}$ \\ Juan José Soler-Cataluña ${ }^{3}$ \\ Joan B Soriano ${ }^{4}$ \\ Marc Miravitlles 5 \\ 'Multimorbidity Patients Unit, \\ Internal Medicine Department, \\ Hospital Universitario Mutua de \\ Terrassa, Universidad de Barcelona, \\ Terrassa, Barcelona, ${ }^{2}$ Department of \\ Pneumology, Hospital Universitario \\ de la Princesa, Universidad Autónoma \\ de Madrid, Madrid, ${ }^{3}$ Department \\ of Pneumology, Hospital Arnau de \\ Vilanova-Lliria, Valencia, ${ }^{4}$ Instituto de \\ Investigación Hospital Universitario \\ de la Princesa (IISP), Universidad \\ Autónoma de Madrid, Madrid, \\ ${ }^{5}$ Department of Pneumology, Vall \\ d'Hebron University Hospital, CIBER \\ de Enfermedades Respiratorias \\ (CIBERES), Barcelona, Spain
}

\begin{abstract}
Current recommendations to consider initiation of palliative care (PC) in COPD patients are often based on an expected poor prognosis. However, this approach is not evidence-based, and which and when COPD patients should start PC is controversial. We aimed to assess whether current suggested recommendations for initiating PC were sufficiently reliable. We identified prognostic variables proposed in the literature for initiating PC; then, we ascertained their relationship with 1-year mortality, and finally, we validated their utility in our cohort of 697 patients hospitalized for COPD exacerbation. From 24 articles of 499 screened, we selected 20 variables and retrieved 48 original articles in which we were able to calculate the relationship between each of them and 1-year mortality. The number of studies where 1-year mortality was detailed for these variables ranged from 9 for previous hospitalizations or $\mathrm{FEV}_{1} \leq 30 \%$ to none for albumin $\leq 25 \mathrm{mg} / \mathrm{dL}$. The percentage of 1-year mortality in the literature for these variables ranged from $5 \%$ to $60 \%$. In the validation cohort study, the prevalence of these proposed variables ranged from $8 \%$ to $64 \%$; only 10 of the 18 variables analyzed in our cohort reached statistical significance with Cox regression analysis, and none overcame an area under the curve $\geq 0.7$. We conclude that none of the suggested criteria for initiating PC based on an expected poor vital prognosis in COPD patients in the short or medium term offers sufficient reliability, and consequently, they should be avoided as exclusive criteria for considering PC or at least critically appraised.
\end{abstract}

Keywords: COPD, palliative care, prognosis, mortality, survival, end of life

\section{Introduction}

COPD is the third leading cause of death worldwide and the seventh in the combination of years of life lost or lived with disability. ${ }^{1}$ Several studies have shown that patients with severe COPD have a similar or greater number of symptoms than those of patients with inoperable lung cancer. ${ }^{2}$ Nonetheless, COPD patients are less likely to receive palliative care (PC) compared with cancer patients. ${ }^{3}$

PC is not limited to the terminal phase of the disease and can be delivered alongside standard therapies, according to the needs and preferences of patients and regardless of the risk of death in the short or medium term. ${ }^{4}$ Traditionally, PC has mainly been used for patients with neoplastic diseases, where it has improved symptoms, quality of life and even survival, but its implementation has also proved effective in several chronic noncancer diseases, including COPD. ${ }^{5}$

One of the main barriers for initiating PC in COPD is the unpredictability of vital prognosis in an individual patient. Many COPD patients present a gradual deterioration, others stability for years, while a subgroup suffers acute COPD exacerbations. Usually, COPD patients are very resilient and survive several of these episodes during their lives, including some severe exacerbations - defined as those requiring hospitalization or even ventilator support - until the patient's death. This uncertain
Correspondence: Marc Miravitlles Department of Pneumology, Hospital Universitari Vall d'Hebron, Pg Vall d'Hebron I19-129, Barcelona 08035, Spain Tel/fax +34932746083 Email mmiravitlles@vhebron.net BY
hereby accept the Terms. Non-commercial uses of the work are permitted without any furcher (unpermission from Dove Medical Press limited, provided the work is properly attributed. For permission hereby accept the Terms. Non-commercial uses of the work are permitted without any further permission from Dove Medical Press Limited, provided the work is properly attributed. For permissio for commercial use of this work, please see paragraphs 4.2 and 5 of our Terms (https://www.dovepress.com/terms.php) 
prognosis complicates matters for clinicians unfamiliar with PC in initiating discussions about PC and end-of-life care planning. ${ }^{6}$ Although many variables have demonstrated a close relationship with impaired survival in COPD, their utility for evaluating survival in an individual patient in the short or medium term is debatable, and therefore, their usefulness as main criteria to initiate PC is not well established.

The main objective of our study was to assess whether current suggested recommendations for PC based on an expected 1-year mortality are sufficiently reliable.

\section{Methods}

This study was structured in 3 phases. 1) To identify variables proposed in the literature as main criteria for considering initiating PC in COPD patients, based on a foreseeable poor prognosis in the short or medium term ( $\leq 1$-year survival). 2) To ascertain the relationship between these proposed variables and 1-year mortality, retrieving the data from the original studies. 3) We sought to validate the utility of the proposed variables in a cohort of patients hospitalized for COPD exacerbation. We followed the PRISMA (preferred reporting items for systematic reviews and meta-analyses) guidance for the systematic review and the TRIPOD (transparent reporting of a multivariable prediction model for individual prognosis or diagnosis) guidance for the validation study.

For this purpose, we conducted a comprehensive search in MEDLINE, using the search strategy detailed in the Supplementary materials. We retrieved and evaluated all publications, including systematic reviews, reviews or expert opinion papers that included variables considered as poor prognostic factors, defined as a projected median survival $\leq 1$ year, as principal criteria for initiating PC. COPD guidelines were also reviewed, selecting those that included recommendations for considering PC based on prognostic variables associated with high probability of death in the coming months. PC guidelines for non-oncological diseases, in which the purpose for initiating PC was based on similar criteria, were added. We also searched the reference lists of identified articles for further relevant papers.

From these reviewed articles, we selected those variables and their respective most often referenced cutoffs as suggested criteria for initiating PC based on a foreseeable poor prognosis in the short or medium term ( $<1$ year). Additionally, we considered 3 multicomponent prognosis indexes (Body mass index, Obstruction, Dyspnea and 6 minutes walking test [BODE], Body mass index, Obstruction, Dyspnea and severe exacerbations [BODEX], and Comorbidity, Obstruction, Dyspnea and severe exacerbations [CODEX]) deemed to be of special interest.
Subsequently, we retrieved all available original articles from which the selection of variables was drawn, and then a new search was performed focussing specifically on each of these individual variables and their relationship with 1-year mortality. Information on 1-year mortality in these articles was extracted when detailed in the text or alternatively if they could be estimated from survival curve figures embedded in the articles (Supplementary materials).

Finally, the reliability of selected variables was validated in a database based on pooled-individual analysis data from 697 patients discharged after a hospitalization for acute exacerbation of COPD and with complete 1-year follow-up for mortality, based on 4 previously published cohorts (Supplementary materials). ${ }^{7-11}$ In order to not duplicate data, these articles were expressly excluded from the previous search. BODE, BODEX and CODEX multicomponent indexes were calculated with the cutoffs previously described in the original studies. ${ }^{12-14}$ (Table S1).

In this database, we specifically analyzed each of the variables suggested in the reviewed articles with the percentages of the population that met each of these factors and their relationship with mortality in the year after hospital discharge, using the cutoffs previously proposed in the literature.

All participants gave their written informed consent to participate, and the ethics committee of Universitari Hospital Mutua de Terrassa approved all previous studies.

\section{Statistical analysis}

Parametric data were expressed as mean and standard deviation (SD) and analyzed with analysis of variance (ANOVA) or Mann-Whitney $U$ test according to the distribution of the variables. Qualitative data were expressed as numbers and percentages and analyzed with the chi-square test. We also calculated the hazard ratio and $95 \%$ confidence intervals for survival time using a Cox logistic regression analysis and Kaplan-Meier survival curves, and statistical significance was calculated with the log-rank test. Receiver operating characteristic (ROC) curves and their area under the curve (AUC) for 1-year mortality were also analyzed separately for qualitative and quantitative variables calculating their sensitivity, specificity and Youden's J Statistic ( J = sensivity + specificity -1). The comparison of AUC was performed with the DeLong method. Statistical significance was established at $P<0.05$.

\section{Results}

For the variables associated with poor prognosis described in the literature as main criteria for initiating $\mathrm{PC}$ in COPD 


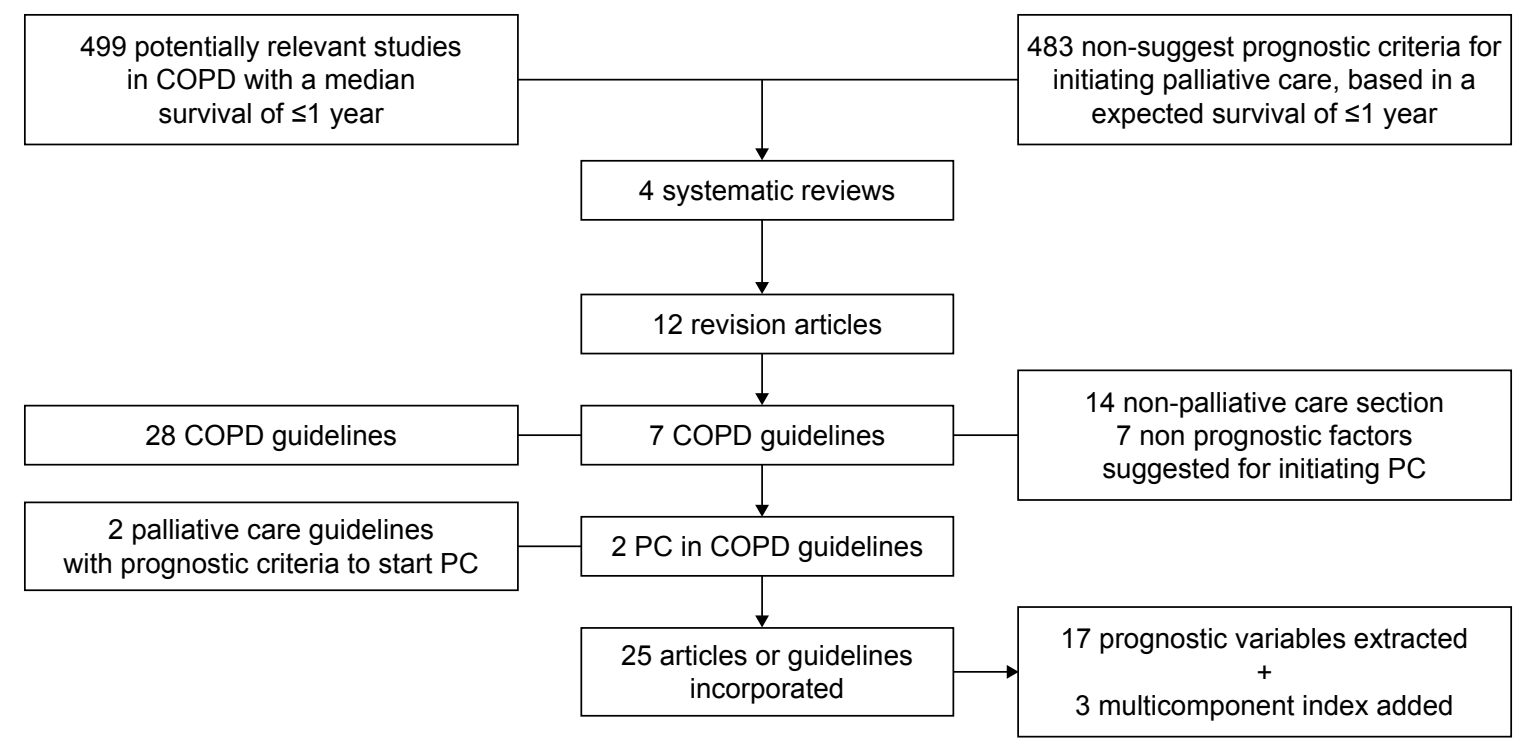

Figure I Flowchart diagram of systematic review.

Abbreviation: PC, palliative care.

patients, we retrieved 499 articles. Of these, in 15 articles -4 systematic reviews and 11 revision articles - several variables considered as useful factors to initiate PC based on a foreseeable 1-year mortality were selected for subsequent analysis. ${ }^{6,15-31}$ Additionally, a total of 28 COPD clinical practice guidelines were reviewed. Of these, 7 recommended various prognostic factors as criteria for starting PC. Another 2 guides of non-oncological PC, based on a similar approach, were also reviewed (Supplementary materials). Altogether, a total of 24 articles were selected, from which 20 variables considered in the bibliography as useful predictors of poor prognosis for considering PC were extracted and analyzed (Figure 1). All revised COPD guidelines and their references are detailed in Table S2.

After searching for previously published original articles on these 20 selected variables and their association with 1 -year mortality, we retrieved 48 original articles in which we were able to calculate the relationship between each of them and the percentage of 1-year mortality. These 20 selected variables, their percentages of 1-year mortality in original articles and number of references, are detailed in Table 1. References of original articles are shown in Table S3.

In Figure 2, the number of studies reviewed for each variable and their mean percentages of 1-year mortality are detailed. As shown in Table 1 and Figure 2, the percentage of 1-year mortality for the proposed variables varies widely between different original articles, reflecting the diverse baseline characteristics of the populations studied. Similarly, the number of original studies with which we were able to calculate 1-year mortality ranged from 9 for hospital admissions in the previous year or $\mathrm{FEV}_{1}(\%)$ to a single study for being married and none for variables such as a plasma albumin level $<25 \mathrm{mg} / \mathrm{dL}$, a criterion proposed by Salpeter et al. ${ }^{16}$

In our validation cohort study, 697 patients hospitalized for COPD exacerbation were included. The mean age of

Table I Variables related with I-year mortality in the systematic review

\begin{tabular}{|c|c|c|c|c|}
\hline Variables & $\mathbf{N}$ & H.M. (\%) & L.M. (\%) & Mean (SD) \\
\hline \multicolumn{5}{|l|}{ Epidemiological } \\
\hline Age $\geq 70$ years & 3 & 51 & 10 & $27(2 I .4)$ \\
\hline Unmarried & I & 5 & 5 & \\
\hline Physical dependence & 1 & 60 & 60 & \\
\hline Poor quality of life & 6 & 27 & 5 & $16.7(7.1)$ \\
\hline \multicolumn{5}{|l|}{ Systemic variables } \\
\hline $\mathrm{BMI} \leq 2 \mathrm{I} \mathrm{kg} / \mathrm{m}^{2}$ & 4 & 56 & 15 & $32.3(19.4)$ \\
\hline Serum albumin $<25 \mathrm{mg} / \mathrm{dL}$ & 0 & & & \\
\hline Comorbidities & 2 & 40 & 8 & $24(22.6)$ \\
\hline Depression & 4 & 25 & 10 & $19.8(6.7)$ \\
\hline Anemia & 1 & 40 & 40 & \\
\hline Cor pulmonale/PH & 3 & 30 & 8 & I5.7 (I2.4) \\
\hline \multicolumn{5}{|l|}{ Respiratory variables } \\
\hline Previous hospitalization & 9 & 42 & 5 & $23.4(12)$ \\
\hline $\mathrm{FEV}_{1} \leq 30 \%$ & 9 & 24 & 4 & $12.6(5.9)$ \\
\hline Dyspnea (mMRC scale) & 2 & 15 & 14 & | $4.5(0.7)$ \\
\hline Hypoxemia & 4 & 35 & 20 & $25.3(6.7)$ \\
\hline Hypercapnia & 3 & 30 & 12 & $20.6(7.4)$ \\
\hline Invasive ventilation & 4 & 54 & 43 & $47.5(4.7)$ \\
\hline NIV & 4 & 48 & 22 & $35.8(\mathrm{II})$ \\
\hline \multicolumn{5}{|l|}{ Multicomponent index } \\
\hline BODE & 5 & 20 & 10 & I $3.8(4.8)$ \\
\hline
\end{tabular}

Notes: N, number of studies retrieved; H.M., higher percentage of I-year mortality in retrieved articles; L.M., lower percentage of I-year mortality in retrieved articles; Mean (SD), mean and standard deviation of I-year mortality in retrieved articles. Abbreviations: PH, pulmonary hypertension; BMl, body mass index; mMRC, modified dyspnea Medical Research Council; NIV, noninvasive ventilation; BODE, Body mass index, Obstruction, Dyspnea and 6 minutes walking test. 


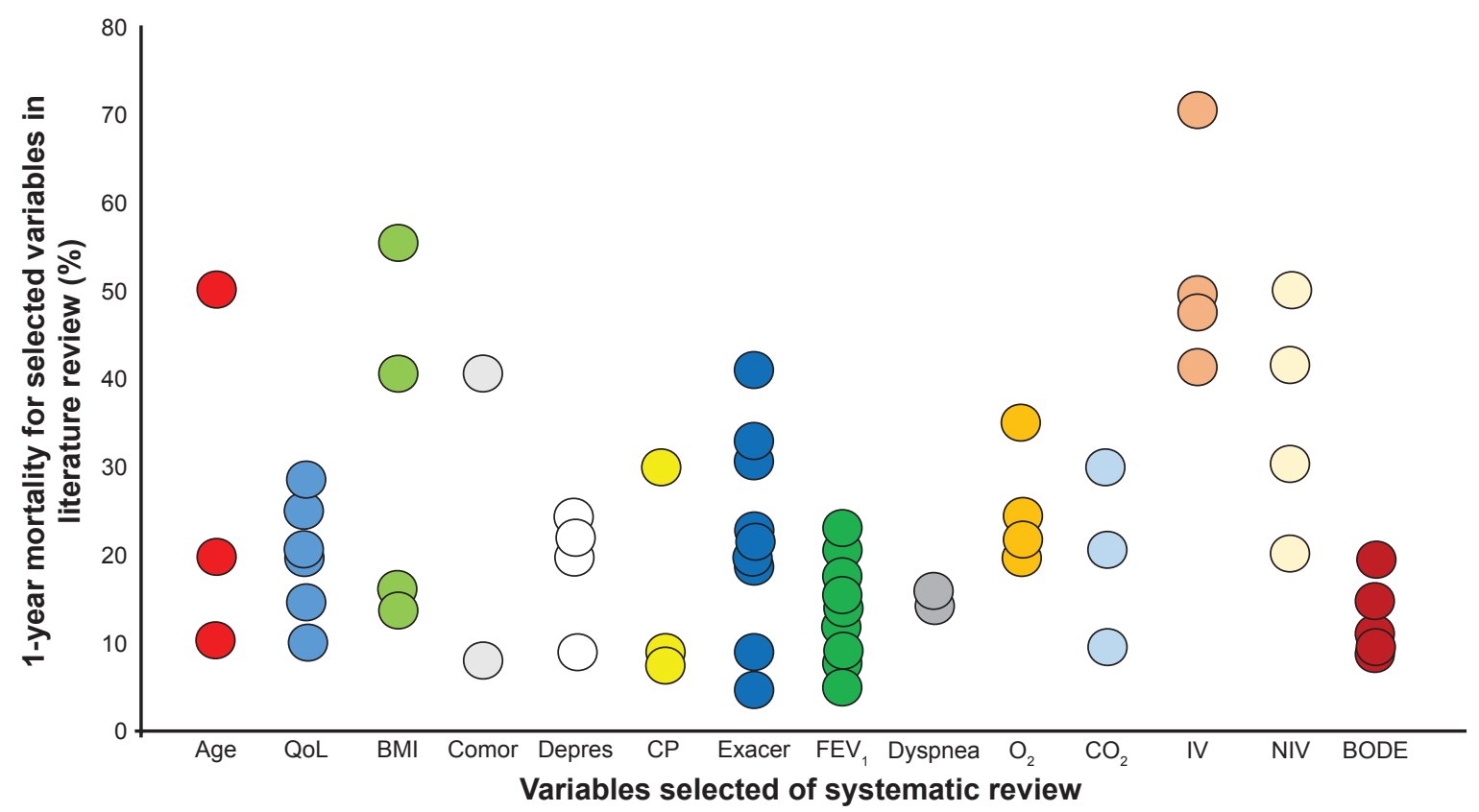

Figure 2 Systematic review, number of studies and I-year mortality.

Notes: The number of circles in each variable represents the number of articles retrieved and their position with respect to $Y$ axis, the mean average mortality. Abbreviations: Exacer, severe exacerbations of COPD in the previous year; Comor, comorbidity; Depres, depression; QoL, quality of life; BMI, body mass index; CP, cor pulmonale; IV, invasive ventilation; NIV, noninvasive ventilation; BODE, Body mass index, Obstruction, Dyspnea and 6 minutes walking test.

the study population was 72.5 years $(\mathrm{SD}=9.5)$, with $93 \%$ males and an overall $17.5 \%$ 1-year mortality. The main characteristics of the study population and their relation with mortality are shown in Table 2. On average, deceased patients were significantly older and had greater functional impairment, more comorbidities, higher levels of depression or dyspnea and more previous admissions for COPD exacerbation (all $P<0.01$ ). Similarly, the scores obtained in

Table 2 Characteristics of the cohort study population and I-year mortality

\begin{tabular}{|c|c|c|c|c|}
\hline Variables & Alive (575) & Dead (122) & Total (697) & $P$-value \\
\hline Age, years (SD) & $71.8(9.4)$ & $75.8(9.3)$ & $72.5(9.5)$ & 0.001 \\
\hline Male (\%) & 539 (94\%) & III (9I\%) & $650(93 \%)$ & 0.32 \\
\hline Smoking (pack-years) (SD) & $57.2(28.5)$ & $61.7(34.4)$ & $57.9(29.5)$ & 0.23 \\
\hline Physical dependence (Katz) (SD) & $5.4(1.1)$ & $5.1(1.2)$ & $5.4(1.2)$ & 0.008 \\
\hline Quality of life (SGRQ) (SD) & $50.5(16)$ & $56.9(19.4)$ & $5 \mathrm{I} .5(16.8)$ & 0.02 \\
\hline Body mass index (SD) & $27.3(4.9)$ & $25.9(4.9)$ & $27(4.9)$ & 0.006 \\
\hline Albumin, mg/dL (SD) & $40.6(4.9)$ & $39.9(4.6)$ & $40.5(4.9)$ & 0.33 \\
\hline Comorbidities (Charlson) (SD) & $2.5(1.6)$ & $3(2)$ & $2.6(1.7)$ & 0.004 \\
\hline Depression (Yesavage) (SD) & $4.5(3.4)$ & $6.2(3.8)$ & $4.8(3.5)$ & 0.001 \\
\hline Cor pulmonale (\%) & $170(30 \%)$ & $43(35 \%)$ & $213(31 \%)$ & 0.23 \\
\hline Hospital admissions in the previous year (SD) & I.4 (I.7) & $1.7(2)$ & I.4 (I.7) & 0.047 \\
\hline FEV,$\%$ post-PBD (SD) & $43.1(15.2)$ & $42(14.9)$ & $42.9(15.2)$ & 0.49 \\
\hline FVC $\%$ post-PBD (SD) & $67(18.6)$ & $64.2(18.1)$ & $66.5(18.5)$ & 0.14 \\
\hline $\mathrm{FEV}_{\mathrm{l}} / \mathrm{FVC}(\mathrm{SD})$ & $49.4(11.2)$ & $49(12.3)$ & $49.4(11.4)$ & 0.67 \\
\hline Dyspnea (mMRC scale) (SD) & $2.3(1.2)$ & $2.7(1.5)$ & $2.4(1.2)$ & 0.001 \\
\hline $\mathrm{PO}_{2}(\mathrm{SD})$ & $61.7(17.3)$ & $60.9(18.5)$ & $61.6(17.5)$ & 0.65 \\
\hline $\mathrm{pCO}_{2}(\mathrm{SD})$ & $45(13)$ & $45.5(15.4)$ & $45(13.4)$ & 0.7 \\
\hline Chronic oxygen therapy (\%) & $159(27.7 \%)$ & 47 (38.5\%) & $206(29.6 \%)$ & 0.02 \\
\hline BODE (SD) & $4.6(2)$ & $5.8(2.6)$ & $4.7(2.1)$ & 0.02 \\
\hline BODEX (SD) & $4.4(2.3)$ & $5.2(2.6)$ & $4.5(2.3)$ & 0.001 \\
\hline CODEX (SD) & $5(2.2)$ & $6.3(2)$ & $5.2(2.2)$ & 0.001 \\
\hline
\end{tabular}

Note: Analysis was performed with ANOVA, Mann-Whitney $U$ test or chi-square test according to the properties of the variables.

Abbreviations: SD, standard deviation; SGRQ, Saint George's Respiratory Questionnaire; mMRC, modified dyspnea Medical Research Council; ANOVA, analysis of variance; BODE, Body mass index, Obstruction, Dyspnea and 6 minutes walking test; BODEX, Body mass index, Obstruction, Dyspnea and severe exacerbations; CODEX, Comorbidity, Obstruction, Dyspnea and severe exacerbations; FVC, forced vital capacity; PBD, postbronchodilatation. 
the BODE, BODEX and CODEX indices were consistently higher for deceased patients (Table 2). The cause of death was respiratory in $51.6 \%$ of cases, cardiovascular in $7.4 \%$, cancer in $6.6 \%$, others in $2.5 \%$ and unknown in $32 \%$ of patients, respectively.

In Table 3, we applied in our cohort for each one of these variables - excluding history of invasive or noninvasive mechanical ventilation and presence of anemia due to lack of data - dichotomized at the commonly proposed cutoffs, alongside with the percentage of population meeting the suggested criteria, the percentage of related 1-year mortality, their sensitivity, specificity and Youden's index. In this table, the statistical significance, hazard ratios, and $95 \%$ confidence intervals were calculated with Cox logistic regression analysis. As detailed in the table, the prevalence of selected variables in our population varied largely. More than half of patients were aged $>70$ years, with scores suggestive of depression in the Yesavage scale ( $\geq 5$ points), and had at least 1 hospitalization during the previous year or dyspnea levels of 3 or 4 on the modified dyspnea Medical Research Council (mMRC) scale. In contrast, severe physical dependence (loss of 3 or more activities of daily living), lower body mass index (BMI; $\leq 21 \mathrm{~kg} / \mathrm{m}^{2}$ ) or serum albumin $<35 \mathrm{mg} / \mathrm{dL}$ were present in $<10 \%$ of patients. Furthermore, the percentage of mortality for the selected variables ranged between $20 \%$ and $30 \%$, and perhaps, $>70 \%$ of patients who met a single poor prognostic criterion were alive at 1 year (Figure 3 ).
Although 10 of the 18 variables analyzed in our cohort reached statistical significance for 1-year mortality, only age $\geq 70$ years, depression (Yesavage scale $\geq 5$ points), health-related quality of life (Saint George's Respiratory Questionnaire $\geq 60$ points) and dyspnea $3-4$ in the mMRC scale had a concomitant sensibility and specificity $\geq 50 \%$. Age $\geq 70$ years had the greatest sensitivity $(0.71)$ with a specificity of 0.41 ; in contrast, the greatest specificity in our cohort was associated with severe functional dependence (0.92) but at the expense of a very low sensitivity (0.11).

In Table 4, the AUC for quantitative variables expressed as continuous (non-tailed) is detailed. The best AUC was obtained by the CODEX index (0.68) and their combination with the Yesavage depression scale (0.69), although without statistical significance versus CODEX alone $(P=0.8)$ (Figure 4). Other variables were not included in this model, since the CODEX index already includes age, comorbidity measured by the Charlson index, $\mathrm{FEV}_{1}(\%)$, dyspnea and hospitalizations in the previous year.

Finally, and given that the models proposed in the literature as main criteria for considering PC based on an expected poor prognosis are usually composed of a combination of variables, we tested an adaptation of the criteria proposed by Curtis et al. ${ }^{15}$ A total of $86 \%$ of patients had 2 or more of these criteria - the cutoff recommended by the author - but the 1-year mortality differences among patients who met $\geq 2$ criteria and the rest did not reach statistical significance $(P=0.83)$. If we use a score $\geq 3$,

Table 3 Predictive mortality variables in the cohort study

\begin{tabular}{|c|c|c|c|c|c|c|c|c|}
\hline Variables & $\%$ & I-year mortality (\%) & Sen & Spe & $Y$ & $P$-value & HR & $95 \% \mathrm{Cl}$ \\
\hline Age $\geq 70$ years & 64 & 20 & 0.74 & 41.6 & 0.16 & 0.001 & 2 & $1.3-3$ \\
\hline Unmarried & 23 & 25 & 0.42 & 0.60 & 0.02 & 0.29 & n.c. & $0.5-1.5$ \\
\hline Physical dependence (loss $\geq 3$ activities of daily living) & 8 & 22 & 0.11 & 0.92 & 0.02 & 0.31 & 1.36 & $0.75-2.48$ \\
\hline Poor quality of life (SGRQ $\geq 6 \mathrm{I}$ ) & 33 & 28 & 0.53 & 0.71 & 0.24 & 0.001 & 2.6 & I.44-4.66 \\
\hline $\mathrm{BMI} \leq 2 \mathrm{I} \mathrm{kg} / \mathrm{m}^{2}$ & 10 & 27 & 0.17 & 0.91 & 0.07 & 0.02 & 1.81 & $1.1-3$ \\
\hline Albumin $\leq 35 \mathrm{mg} / \mathrm{dL}$ & 10 & 33 & 0.19 & 0.92 & 0.11 & 0.03 & 2.2 & $1.6-4.5$ \\
\hline Comorbidities (Charlson $\geq 3$ ) & 23 & 22 & 0.28 & 0.78 & 0.07 & 0.07 & 1.4 & $0.97-2.14$ \\
\hline Depression (Yesavage $\geq 5$ points) & 57 & 27 & 0.61 & 0.61 & 0.21 & 0.003 & 2.24 & $1.32-3.8$ \\
\hline Cor pulmonale & 31 & 21 & 0.35 & 0.7 & 0.05 & 0.28 & 1.2 & $0.85-1.8$ \\
\hline$\geq I$ hospitalization in the previous year & 63 & 18 & 0.68 & 0.47 & 0.15 & 0.24 & 1.27 & $0.87-1.84$ \\
\hline Chronic oxygen therapy & 30 & 22 & 0.39 & 0.72 & 0.11 & 0.01 & 1.6 & $1.1-2.3$ \\
\hline $\mathrm{FEV}_{1} \leq 30 \%$ & 22 & 19 & 0.25 & 0.78 & 0.3 & 0.5 & 1.12 & $0.74-1.69$ \\
\hline Dyspnea (mMRC scale $\geq 3$ ) & 55 & 24 & 0.59 & 0.57 & 0.16 & 0.002 & 1.8 & $1.25-2.61$ \\
\hline $\mathrm{pO}_{2} \leq 50 \mathrm{mmHg}$ & 21 & 21 & 0.28 & 0.77 & 0.06 & 0.1 & 1.33 & $0.88-2.1$ \\
\hline $\mathrm{PCO}_{2} \geq 50 \mathrm{mmHg}$ & 26 & 21 & 0.31 & 0.75 & 0.06 & 0.2 & 1.3 & $0.87-1.94$ \\
\hline $\mathrm{BODE} \geq 7$ & 21 & 24 & 0.47 & 0.82 & 0.29 & 0.007 & 3.74 & I.4-9.7 \\
\hline BODEX $\geq 7$ & 18 & 24 & 0.21 & 0.84 & 0.05 & 0.07 & 1.49 & $0.97-2.3$ \\
\hline CODEX $\geq 7$ & 21 & 29 & 0.49 & 0.73 & 0.22 & 0.001 & 2.42 & $1.7-3.5$ \\
\hline
\end{tabular}

Notes: \%, percentage of the population with the variable criteria. Statistical significance, HRs, and $95 \%$ Cls were calculated with Cox logistic regression analysis. Abbreviations: HR, hazard ratio; Cl, confidence interval; SGRQ, Saint George's Respiratory Questionnaire; BMI, body mass index; mMRC, modified dyspnea Medical Research Council; Sen, sensitivity; Spe, specificity; Y, Youden's index; n.c., non calculated; BODE, Body mass index, Obstruction, Dyspnea and 6 minutes walking test; BODEX, Body mass index, Obstruction, Dyspnea and severe exacerbations; CODEX, Comorbidity, Obstruction, Dyspnea and severe exacerbations. 

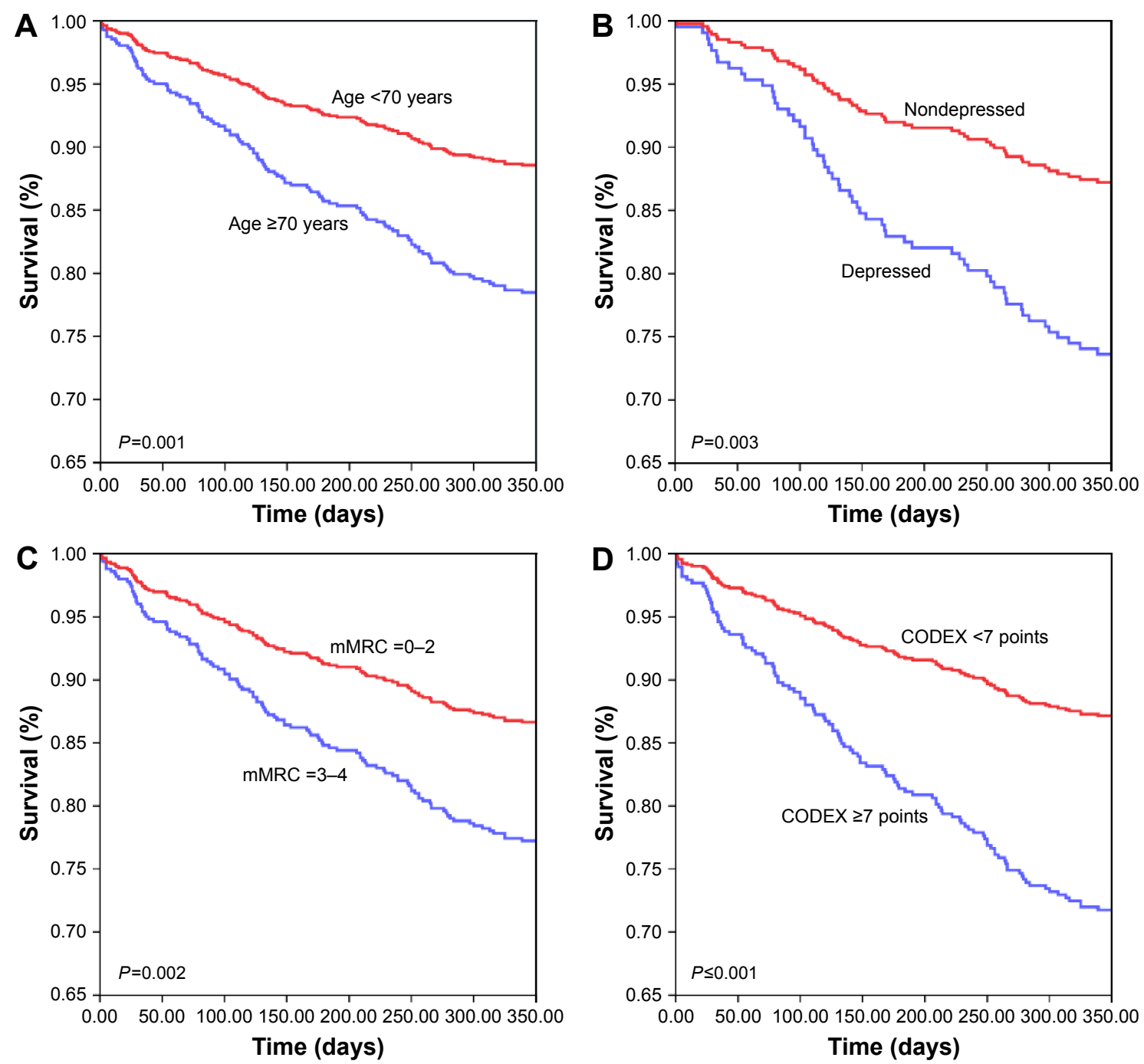

Figure 3 Kaplan-Meier curves for (A) age, (B) depression, (C) dyspnea, and (D) CODEX index.

Abbreviations: mMRC, modified dyspnea Medical Research Council; CODEX, Comorbidity, Obstruction, Dyspnea and severe exacerbations.

Table 4 AUC for selected variables in the cohort study

\begin{tabular}{|c|c|c|c|}
\hline Variables & AUC & $P$-value & $95 \% \mathrm{Cl}$ \\
\hline Age, years & 0.62 & 0.0001 & $0.58-0.65$ \\
\hline Physical dependence (Katz index) & 0.59 & 0.0005 & $0.55-0.62$ \\
\hline Quality of life (SGRQ) & 0.52 & 0.01 & $0.48-0.55$ \\
\hline BMI & 0.59 & 0.001 & $0.55-0.63$ \\
\hline Albumin & 0.5 & 0.9 & $0.46-0.54$ \\
\hline Comorbidities (Charlson index) & 0.56 & 0.02 & $0.52-0.6$ \\
\hline Depression (Yesavage scale) & 0.63 & 0.001 & $0.57-0.68$ \\
\hline $\mathrm{FEV}_{1}$ & 0.52 & 0.5 & $0.48-0.55$ \\
\hline Dyspnea & 0.58 & 0.002 & $0.55-0.62$ \\
\hline $\mathrm{pO}_{2}, \mathrm{mmHg}$ & 0.52 & 0.52 & $0.48-0.57$ \\
\hline $\mathrm{pCO}_{2}, \mathrm{mmHg}$ & 0.52 & 0.58 & $0.48-0.55$ \\
\hline BODE & 0.54 & 0.05 & $0.49-0.57$ \\
\hline BODEX & 0.59 & 0.001 & $0.55-0.63$ \\
\hline CODEX & 0.68 & 0.0001 & $0.62-0.72$ \\
\hline Curtis criteria & 0.64 & 0.0001 & $0.6-0.68$ \\
\hline
\end{tabular}

Abbreviations: AUC, area under the curve; $\mathrm{Cl}$, confidence interval; SGRQ, Saint George's Respiratory Questionnaire; BMI, body mass index; BODE, Body mass index, Obstruction, Dyspnea and 6 minutes walking test; BODEX, Body mass index, Obstruction, Dyspnea and severe exacerbations; CODEX, Comorbidity, Obstruction, Dyspnea and severe exacerbations.
$61 \%$ of patients met the criteria, with statistical significance $(P<0.0001$; HR: 2.49 ; 95\% CI: $1.68-3.7)$ and an AUC of $0.64(0.6-0.68)$. In contrast, the criteria suggested by Salpeter are so restrictive that none of the patients in our cohort fulfilled the proposed criteria, even after changing the cutoffs of albumin from 25 to $35 \mathrm{mg} / \mathrm{dL}$ and BMI from 18 to $21 \mathrm{~kg} / \mathrm{m}^{2} \cdot{ }^{16}$

\section{Discussion}

The main conclusion of our study is that none of the suggested criteria for initiating PC based on an expected poor vital prognosis in COPD patients in the short or medium term offers sufficient reliability, and perhaps, they should be avoided as exclusive criteria for considering PC or at least critically evaluated.

Obviously, this does not mean that these proposed variables and multicomponent indices are not useful 


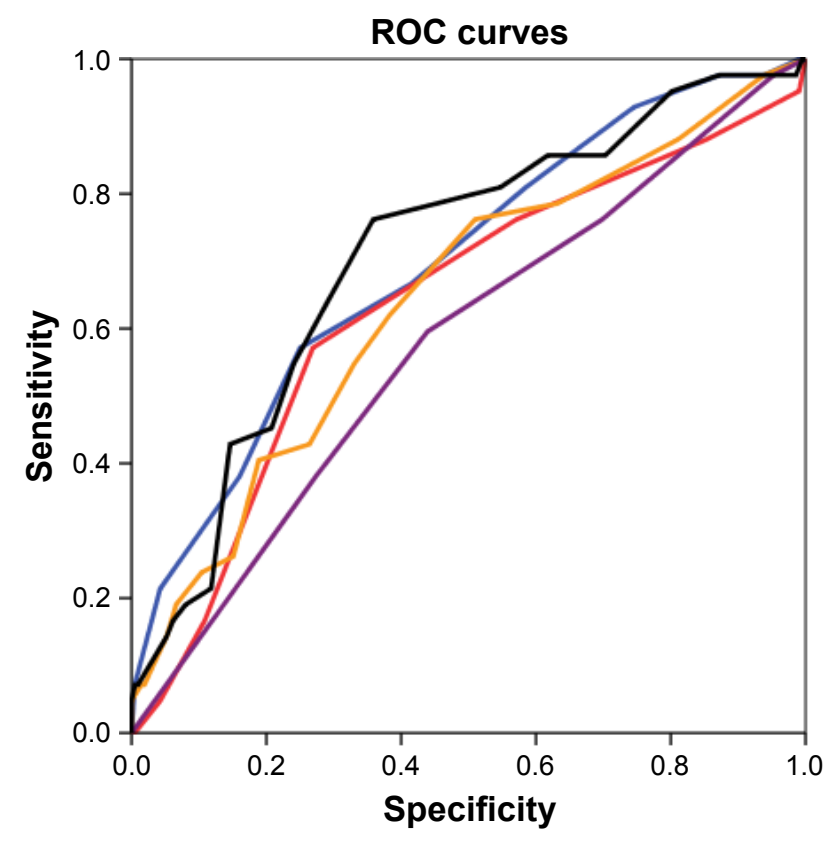

Figure 4 ROC curves.

Notes: Black line, CODEX + Yesavage; blue line, CODEX; orange line, Yesavage; red line, Curtis criteria; violet line, dyspnea.

Abbreviations: ROC, receiver operating characteristic; CODEX, Comorbidity, Obstruction, Dyspnea and severe exacerbations.

predictors of survival in COPD, but as we show in our study, their applicability to individual patients at short or medium term is limited. This is because these prognostic factors are usually related with long-term survival (3-10 years) and their utility has been explored in diverse populations, and they are difficult to apply in any particular patient. For example, the most widely used criterion for evaluating COPD severity is the postbronchodilator $\mathrm{FEV}_{1}(\%)$, which is clearly related with survival. This was again confirmed in a recent study (COCOMICS) performed in a large pooled analysis of COPD patients. In this study, patients with an $\mathrm{FEV}_{1}(\%)<35 \%$ had a 6 -fold greater 5-year mortality than the group with an $\mathrm{FEV}_{1} \geq 75 \%$. However, the probability of survival for patients with $\mathrm{FEV}_{1}(\%) \leq 35 \%$ was $46 \%$ at 5 years. ${ }^{32}$ Moreover, in this study, $\mathrm{FEV}_{1}(\%)$ was a better predictor of survival than dyspnea measured with the mMRC scale, contrary to Nishimura et $\mathrm{al}^{33}$ and even to this cohort. The most plausible explanation is the broad spectrum of patients included in the COCOMICS study, while in the Nishimura et al's study and in this cohort, patients included had more severe COPD, suggesting that in patients with advanced COPD, dyspnea discriminates better than $\mathrm{FEV}_{1}(\%)$.

Furthermore, these criteria vary widely according to the population studied. For example, a BODE score from 7 to 10 , proposed as a criterion for considering PC in COPD patients in several publications, carries a year mortality ranging from $7 \%$ in the original cohort, based on ambulatory patients without comorbidity, to $>20 \%$ in patients hospitalized for COPD exacerbation. ${ }^{10,34}$ Of note, the highest percentages of mortality for age or BMI in the literature review were obtained from the study of Connors et al. ${ }^{35}$ This study is part of the SUPPORT project - conducted in patients with a life expectancy of $<6$ months for several chronic diseases - and in it COPD includes only patients hospitalized with hypercapnic respiratory failure and without spirometric confirmation. ${ }^{36}$

Another of the proposed approaches for considering PC in chronic illness, and not analyzed in our cohort due to lack of data, is the so-called surprise question: "Would I be surprised if my patient were to die in the next 12 months?" However, its application in COPD has proven highly imprecise. Thus, in the SUPPORT study, the interviews were performed with physicians 5 days before the deaths of COPD patients, and the doctors predicted an expected survival probability of $50 \%$ at 6 months.${ }^{37}$ By contrast, in patients hospitalized in intensive care units (ICUs) for COPD or asthma, physicians estimated that only $10 \%$ of patients would survive $>6$ months, compared with the $40 \%$ observed during follow-up. ${ }^{38}$

Similarly, a criterion widely suggested as a useful predictor of short- or medium-term mortality in chronic diseases is the progressive physical functional dependence. Several authors believe that in patients with severe COPD, the last year of life is characterized by a gradual dependence in daily living activities that worsens with each exacerbation. Although it is well known that severe COPD exacerbations cause a decrease of physical activity, in most of the decedents for chronic diseases, the course of disability in the last year of life did not follow a predictable pattern, and individual trajectories are highly variable. ${ }^{39}$

In our opinion, reinforced by the data of this study, the decision to start PC - together with restorative care should be based on the presence of symptoms refractory to conventional therapy, alongside the preferences of patients. PC includes, among other elements, care planning communication, end-of-life decisions, limitation of aggressive treatments (ICU admission, mechanical ventilation and cardiopulmonary resuscitation) and symptomatic treatment, while always considering the physical, psychosocial and spiritual aspects and preferences of patients..$^{40}$ Regrettably, PC is often erroneously considered restricted to terminal care, when it has been shown to be applicable and of value in other stages of COPD. ${ }^{41}$ It does not imply abandoning other therapies, and as shown in our article, it is not limited by the prognostic uncertainty inherent to COPD. Several studies have shown that mortality in COPD is highly concentrated in the days or weeks following an exacerbation and frequently related with comorbidities, and therefore hardly predictable. ${ }^{11,42}$ 
Our study highlights the importance of symptoms such as dyspnea and depression in patients hospitalized for COPD, not only for their high prevalence and prognostic implications but also for their impact on the quality of life of these patients. These symptoms can be improved by PC alongside the usual medical therapies, non-pharmacological measures and psychological treatment.

Our study has some limitations. First, in the search of original articles, only publications in which we were able to extract the percentage of 1 -year mortality were included. That is, if a study examined the relationship between a prognostic variable and 3-year survival, but 1-year mortality was not detailed in the text or readily calculated from the survival curves, it was not included. Second, the analyzed cohorts included only patients hospitalized for COPD exacerbation, among whom mortality is generally higher than that in ambulatory patients. However, we believe that our findings are applicable to outpatients with severe COPD with a history of previous admissions. Finally, like other COPD studies performed in Spain, the percentage of females included in our study is small, and we were unable to explore the gender differences.

In conclusion our study demonstrates that none of the suggested criteria for initiating PC in COPD patients based on an expected poor vital prognosis in the short or medium term offers sufficient reliability, and perhaps, the initiation of PC in COPD patients should not be based on life expectancy, since this is extremely imprecise for any given individual patient.

\section{Disclosure}

The authors report no conflicts of interest in this work.

\section{References}

1. Global Burden of Disease (GBD) [webpage on the Internet]. Institute for Health Metrics and Evaluation. Available from: http://www.healthdata. org/gbd. Accessed February 23, 2017.

2. Weingaertner V, Scheve C, Gerdes V, et al. Breathlessness, functional status, distress, and palliative care needs over time in patients with advanced chronic obstructive pulmonary disease or lung cancer: a cohort study. J Pain Symptom Manage. 2014;48(4):569-581.

3. Au DH, Udris EM, Fihn SD, McDonell MB, Curtis JR. Differences in health care utilization at the end of life among patients with chronic obstructive pulmonary disease and patients with lung cancer. Arch Intern Med. 2006;166(3):326-331.

4. World Health Organization. WHO Definition of Palliative Care. Who.int. Available from: www.who.int/cancer/palliative/definition/en/. Accessed April 4, 2017.

5. Higginson IJ, Bausewein C, Reilly CC, et al. An integrated palliative and respiratory care service for patients with advanced disease and refractory breathlessness: a randomised controlled trial. Lancet Respir Med. 2014;2(12):979-987.
6. Duenk RG, Heijdra Y, Verhagen SC, Dekhuijzen RP, Vissers KC, Engels Y. PROLONG: a cluster controlled trial to examine identification of patients with COPD with poor prognosis and implementation of proactive palliative care. BMC Pulm Med. 2014;14:54.

7. Almagro P, Calbo E, Ochoa de Echagüen A, et al. Mortality after hospitalization for COPD. Chest. 2002;121(5):1441-1448.

8. Sanjaume M, Almagro P, Rodríguez-Carballeira M, Barreiro B, Heredia JL, Garau J. Post-hospital mortality in patients re-admitted due to COPD. Utility of BODE index. Rev Clin Esp. 2009;209(8): 364-370.

9. Almagro P, Salvadó M, Garcia-Vidal C, et al. Recent improvement in long-term survival after a COPD hospitalisation. Thorax. 2010; 65(4):298-302.

10. Almagro P, Salvadó M, Garcia-Vidal C, et al. Pseudomonas aeruginosa and mortality after hospital admission for chronic obstructive pulmonary disease. Respiration. 2012;84(1):36-43.

11. Almagro P, Cabrera FJ, Diez J, et al. Comorbidities and short-term prognosis in patients hospitalized for acute exacerbation of COPD: the EPOC en Servicios de medicina interna (ESMI) study. Chest. 2014;142(5): 1126-1133.

12. Celli BR, Cote CG, Marin JM, et al. The body-mass index, airflow obstruction, dyspnea, and exercise capacity index in chronic obstructive pulmonary disease. $N$ Engl J Med. 2004;350(10):1005-1012.

13. Soler-Cataluña JJ, Martínez-García MA, Sánchez LS, Tordera MP, Sánchez PR. Severe exacerbations and BODE index: two independent risk factors for death in male COPD patients. Respir Med. 2009; 103(5):692-699.

14. Almagro P, Soriano JB, Cabrera FJ; Working Group on COPD, Spanish Society of Internal Medicine. Short- and medium-term prognosis in patients hospitalized for COPD exacerbation: the CODEX index. Chest. 2014;145(5):972-980.

15. Curtis JR. Palliative and end-of-life care for patients with severe COPD. Eur Respir J. 2008;32(3):796-803.

16. Salpeter SR, Luo EJ, Malter DS, Stuart B. Systematic review of noncancer presentations with a median survival of 6 months or less. $\mathrm{Am} \mathrm{J}$ Med. 2012;125(5):512.e1-512.e6.

17. Coventry PA, Grande GE, Richards DA, Todd CJ. Prediction of appropriate timing of palliative care for older adults with non-malignant life-threatening disease: a systematic review. Age Ageing. 2005;34(3): 218-227.

18. Singanayagam A, Schembri S, Chalmers JD. Predictors of mortality in hospitalized adults with acute exacerbation of chronic obstructive pulmonary disease. A systematic review and meta-analysis. Ann Am Thorac Soc. 2013;10(2):81-89.

19. Van Mechelen W, Aertgeerts B, De Ceulaer K. Defining the palliative care patient: a systematic review. Palliat Med. 2013;27(3):197-208.

20. Weissman DE, Meier DE. Identifying patients in need of a palliative care assessment in the hospital setting: a consensus report from the Center to Advance Palliative Care. J Palliat Med. 2011;14(1): $17-23$.

21. Berman AR. Management of patients with end-stage chronic obstructive pulmonary disease. Prim Care. 2011;38(2):277-297.

22. Hardin KA, Meyers F, Louie S. Integrating palliative care in severe chronic obstructive lung disease. COPD. 2008;5(4):207-220.

23. Lal AA, Case AA. Palliation of chronic obstructive pulmonary disease. Ann Palliat Med. 2014;3(4):276-285.

24. Thoonsen B, Groot M, Engels Y, et al. Early identification of and proactive palliative care for patients in general practice, incentive and methods of a randomized controlled trial. BMC Fam Pract. 2011;12:123.

25. Hansen-Flaschen J. Chronic obstructive pulmonary disease: the last year of life. Respir Care. 2004;49(1):90-97.

26. Escarrabill J, Soler Cataluña JJ, Hernández C, Servera E. Recommendations for end-of-life care in patients with chronic obstructive pulmonary disease. Arch Bronconeumol. 2009;45(6):297-303.

27. Carlucci A, Guerrieri A, Nava S. Palliative care in COPD patients: is it only an end-of-life issue? Eur Respir Rev. 2012;21(126):347-354. 
28. Halpin DM, Seamark CJ, Seamark DA. End-of-life care for patients with COPD in the community setting. Br J Gen Pract. 2008;58(551): 390-392.

29. Patel K, Janssen DJ, Curtis JR. Advance care planning in COPD. Respirology. 2012;17(1):72-78.

30. Rocker GM, Sinuff T, Horton R, Hernandez P. Advanced chronic obstructive pulmonary disease: innovative approaches to palliation. J Palliat Med. 2007;10(3):783-797.

31. Szekendi MK, Vaughn J, Lal A, Ouchi K, Williams MV. The prevalence of inpatients at thirty-three U.S. hospitals appropriate for and receiving referral to palliative care. J Palliat Med. 2016;19(4):360-372.

32. Almagro P, Martinez-Camblor P, Soriano JB, et al. Finding the best thresholds of FEV1 and dyspnea to predict 5-year survival in COPD patients: the COCOMICS study. PLoS One. 2014;9(2):e89866.

33. Nishimura K, Izumi T, Tsukino M, Oga T. Dyspnea is a better predictor of 5-year survival than airway obstruction in patients with COPD. Chest. 2002;121(5):1434-1440.

34. Ko FW, Tam W, Tung AH, et al. A longitudinal study of serial BODE indices in predicting mortality and readmissions for COPD. Respir Med. 2011;105(2):266-273.

35. Connors AF Jr, Dawson NV, Thomas C. Outcomes following acute exacerbation of severe chronic obstructive lung disease. The SUPPORT investigators (Study to Understand Prognoses and Preferences for Outcomes and Risks of Treatments). Am J Respir Crit Care Med. 1996; 154(4):959-967.
36. A controlled trial to improve care for seriously ill hospitalized patients. The study to understand prognoses and preferences for outcomes and risks of treatments (SUPPORT). The SUPPORT Principal Investigators. JAMA. 1995;274(20):1591-1598.

37. Fox E, Landrum-McNiff K, Zhong Z, Dawson NV, Wu AW, Lynn J. Evaluation of prognostic criteria for determining hospice eligibility in patients with advanced lung, heart, or liver disease. SUPPORT Investigators. Study to Understand Prognoses and Preferences for Outcomes and Risks of Treatments. JAMA. 1999;282(17):1638-1645.

38. Wildman MJ, Sanderson C, Groves J, et al. Implications of prognostic pessimism in patients with chronic obstructive pulmonary disease (COPD) or asthma admitted to intensive care in the UK within the COPD and asthma outcome study (CAOS): multicentre observational cohort study. BMJ. 2007;335(7630):1132.

39. Gill TM, Gahbauer EA, Han L, Allore HG. Trajectories of disability in the last year of life. N Engl J Med. 2010;362(13):1173-1180.

40. Vermylen JH, Szmuilowicz E, Kalhan R. Palliative care in COPD: an unmet area for quality improvement. Int J Chron Obstruct Pulmon Dis. 2015;10:1543-1551.

41. Duenk RG, Verhagen C, Dekhuijzen P, Vissers K, Engels Y, Heijdra Y. The view of pulmonologists on palliative care for patients with COPD: a survey study. Int J Chron Obstruct Pulmon Dis. 2017;12:299-311.

42. Suissa S, Dell'Aniello S, Ernst P. Long-term natural history of chronic obstructive pulmonary disease: severe exacerbations and mortality. Thorax. 2012;67(11):957-963.
International Journal of COPD

\section{Publish your work in this journal}

The International Journal of COPD is an international, peer-reviewed journal of therapeutics and pharmacology focusing on concise rapid reporting of clinical studies and reviews in COPD. Special focus is given to the pathophysiological processes underlying the disease, intervention programs, patient focused education, and self management protocols.

\section{Dovepress}

This journal is indexed on PubMed Central, MedLine and CAS. The manuscript management system is completely online and includes a very quick and fair peer-review system, which is all easy to use. Visit http://www.dovepress.com/testimonials.php to read real quotes from published authors. 